\title{
Effects of menopause on corneal topography and dry eye
}

\author{
Fethiye Gulden Turgut ${ }^{1}$, Abdulkadir Turgut ${ }^{2 *}$, Zehra Nihal Dolgun ${ }^{3}$, Nadiye Köroğlu ${ }^{4}$
}

\begin{abstract}
${ }^{1}$ Department of Opthalmology, ${ }^{2}$ Department of Obstetrics and Gynecology, Istanbul Medeniyet University Goztepe Training and Research Hospital, Istanbul, Turkey

${ }^{3}$ Department of Obstetrics and Gynecology, Trakya University of Faculty of Medicine, Istanbul, Turkey

${ }^{4}$ Department of Obstetrics and Gynecology, Kanuni Sultan Suleyman Training and Research Hospital, Istanbul, Turkey
\end{abstract}

Received: 15 December 2016

Accepted: 07 January 2017

\author{
*Correspondence: \\ Dr. Abdulkadir Turgut, \\ E-mail: abdulkadirturgut@gmail.com
}

Copyright: ( $)$ the author(s), publisher and licensee Medip Academy. This is an open-access article distributed under the terms of the Creative Commons Attribution Non-Commercial License, which permits unrestricted non-commercial use, distribution, and reproduction in any medium, provided the original work is properly cited.

\begin{abstract}
Background: The aim of this study was to investigate the effect of estrogen and progesterone on central corneal thickness, corneal curvature, and the development of dry eye in postmenopausal women.

Methods: This is a case-control study including 54 premenopausal and 54 postmenopausal women. The two groups were compared in terms of follicle stimulating hormone, estradiol, progesterone, and horizontal and vertical curvatures, astigmatism, central corneal thickness, Schirmer I test scores, and dry eye scores of both eyes.

Results: In the postmenopausal women, central corneal thickness was significantly thinner in both eyes $(p=0.017)$ and the Schirmer test scores were significantly lower both for the right $(\mathrm{p}=0.001)$ and the left eye $(\mathrm{p}=0.003)$. Estradiol levels were positively correlated with central corneal thickness $(\mathrm{p}=0.003-0.006)$ and Schirmer test $\mathrm{scores}(\mathrm{p}=0.004-$ 0.002) whereas progesterone levels showed a positive correlation only with Schirmer test scores $(\mathrm{p}=0.036-0.044)$. Although a significant positive correlation was found between estradiol levels and dry eye scores $(p=0,009)$, no significant correlation was found between progesterone levels and dry eye scores $(p=0,118)$.

Conclusions: Age-related hormonal abnormalities lead to central corneal thinning, increased dry eye symptoms, and decreased tear flow, particularly in the postmenopausal term. Decreased estrogen levels are associated with central corneal thickness and dry-eye symptoms, decreased estrogen and progesterone levels correlates well with diminished tear production but progesterone levels does not effect dry-eye symptoms.
\end{abstract}

Keywords: Corneal topography, Dry eye, Menopause

\section{INTRODUCTION}

The structure and functions of the cornea and the lacrimal and meibomian glands are under the influence of sex hormones and may be affected by sex-based differences. Although the exact effect mechanism of sex steroid hormones remains unknown, this effect is considered to be associated with the presence of estrogen, progesterone, and androgen receptors in the human corneal epithelium, stromal and endothelial cell nuclei, lacrimal and meibomian glands, and palpebral and bulbar conjunctiva. $^{1-3}$
Dry eye syndrome (DES) is a common disorder of the tear film and the ocular surface and affects $2-15 \%$ of the general population. ${ }^{4,5}$ According to the International Dry Eye Workshop in 2007, DES is defined as "a multifactorial disease of tears and ocular surface that results in symptoms of discomfort, visual disturbance, and tear film instability with potential damage to the ocular surface. It is accompanied by increased osmolarity of the tear film and inflammation of the ocular surface". 6 The hormonal change in the menstrual cycle and postmenopausal term have been reported to be related to 
ocular surface dryness and tear production similar with the findings of DES. ${ }^{7-8}$

In this study, depending on the fact that the tear film and the structure of the cornea are the major components of the vision and are influenced by sex hormones, predominantly by estrogen, we aimed to investigate the effect of diminished estrogen, and progesterone, which has been rarely studied in the literature, on central corneal thickness (CCT), corneal curvature, and the development of dry eye symptoms in postmenopausal women and to compare with these parameters with premenopausal women and their hormonal levels.

\section{METHODS}

This case-control study included a total of 108 female patients comprised of 54 premenopausal and 54 postmenopausal women between January 2010 and May 2016. The study was performed in accordance with the Helsinki Declaration and was approved by the Local Ethics Committee.

The groups were compared in terms of age (years), demographic and ocular history, anterior and posterior segment examinations, FSH ( $\mathrm{mIU} / \mathrm{ml}), \mathrm{LH}(\mathrm{mIU} / \mathrm{ml})$, estradiol $(\mathrm{pg} / \mathrm{ml})$, progesterone $(\mathrm{ng} / \mathrm{ml})$, fasting blood glucose (FBG) (mg/dl), thyroid-stimulating hormone (TSH) (uIU/ml), the horizontal and vertical curvatures of both eyes (diopter (D) (degrees), CCT measurements $(\mu \mathrm{m})$, intraocular pressure (IOP) $(\mathrm{mmHG})$, Schirmer I test (ST 1) scores, and dry eye scores.

Corneal curvature was measured by Topcon keratometry. IOP was measured on an air-puff tonometry (AP) (Reichert 7). Optical pahcymetry was used to measure CCT on a non-contact microscope (Topcon, SP3000P).

Dry eye was evaluated by dry eye symptom scoring and non-anesthetic Schirmer test. The symptoms were scored as follows: 0: no symptoms presented (symptoms including ocular dryness, burning, foreign body sensation, redness in the eyes, blurred vision, watery eyes, eye irritation when reading or driving, ocular pain when waking, photophobia, and sensitivity against environmental factors such as air conditioner or windy conditions); 1: episodic symptoms; 2: frequent symptoms; 3: persistent symptoms. Schirmer I test without topical anesthesia was performed using a standard Schirmer paper strip (Whatman filter paper strip measuring $5 \times 35 \mathrm{~mm}$ ).

Hormone levels were measured in the luteal phase for the premenopausal women. The study excluded the patients with contact lenses, prior ocular surgery or a history of trauma, inflammatory ocular disease, diabetes mellitus, hormone replacement therapy, ocular surface disease, a history of topical ophthalmic drugs, and systemic diseases or a history of treatment for systemic diseases.

\section{Statistical analysis}

Data were analyzed using SPSS 23.0 for Windows (SPSS, Inc., Chicago, IL, USA). Normal distribution of data was tested using the Shapiro-Wilk test. MannWhitney-U test was used for the comparison of nonparametric variables. The relationship between estrogen, progesterone levels and other variables was evaluated using the Spearman correlation coefficient. The relationship between the estogen, progesterone levels and the dry eye scores was evaluated by using the Kruskal Wallis H-Test. Results were expressed as mean \pm standard deviation (SD). A p value of $\leq 0.05$ was accepted as statistically significant.

\section{RESULTS}

Table 1 presents the demographic, refractive and dry eye findings of the pre- and post-menopausal women. In the postmenopausal group, CCT was significantly thinner in both eyes $(p=0.017)$ and the ST 1 scores were significantly lower both for the right $(\mathrm{p}=0.001)$ and the left eye $(\mathrm{p}=0.003)$ compared to premenopausal group.

Table 2 presents the correlation between estradiol levels and horizontal and vertical curvatures of eyes, astigmatism values, CCT values, and ST 1 scores. Estradiol levels established a positive correlation with right eye CCT $(r=0.286, p=0.003)$, left eye CCT $(\mathrm{r}=0.261, \mathrm{p}=0.006)$, right eye ST 1 scores $(\mathrm{r}=0.279$, $\mathrm{p}=0.004)$, and left eye ST 1 scores $(\mathrm{r}=0.298, \mathrm{p}=0.002)$.

Table 3 presents the correlation between the progesterone levels and horizontal and vertical curvatures, astigmatism values, CCT values, and ST I scores of both eyes. The progesterone levels established a positive correlation with right eye ST 1 scores $(\mathrm{r}=0.202, \mathrm{p}=0.036)$ and left eye Schirmer test scores $(\mathrm{r}=0.194, \mathrm{p}=0.044)$.

The relationship between the estradiol and progesterone levels and the dry eye scores was evaluated by using the Kruskal Wallis H-Test. A significant positive correlation was found between the estrogen levels and the dry eye scores $(\mathrm{p}=0.009)$, whereas no significant correlation was found between the progesterone levels and the dry eye scores $(\mathrm{p}=0.118)($ Table 4$)$.

\section{DISCUSSION}

Menopausal period, which is characterized by aging and loss of sex steroid hormones, leads to various changes in two major components of the visual system, the cornea and the ocular surface. These changes may result in DES that affects the ocular surface and its glands, thereby leading to tear film alterations, discomfort, and decreased vision, which affect quality of life. ${ }^{9}$

Measurement of corneal thickness has recently gained remarkable importance in clinical practice 
Table 1: Demographic, refractive and dry eye findings in premenopausal and postmenopausal women.

\begin{tabular}{|c|c|c|c|}
\hline Parameters & $\begin{array}{l}\text { Premenopausal }(\mathrm{n}=54) \\
(\text { Mean } \pm \text { SD })\end{array}$ & $\begin{array}{l}\text { Postmenopausal }(\mathrm{n}=54) \\
\text { (Mean } \pm \text { SD) }\end{array}$ & $\mathbf{p}$ \\
\hline Age (years) & $37.24 \pm 7.88$ & $58.02 \pm 8.72$ & 0.000 \\
\hline FSH (mIU/ml) & $6.84 \pm 3.7$ & $77.22 \pm 26.45$ & 0.000 \\
\hline $\mathrm{LH}(\mathrm{mIU} / \mathrm{ml})$ & $6.81 \pm 3.15$ & $35.84 \pm 16.38$ & 0.000 \\
\hline Estradiol (pg/ml) & $102.31 \pm 71.03$ & $11.75 \pm 8.94$ & 0.000 \\
\hline Progesterone (ng/ml) & $6.17 \pm 5.8$ & $0.47 \pm 0.36$ & 0.000 \\
\hline FBG $(\mathrm{mg} / \mathrm{dl})$ & $93.46 \pm 9.83$ & $94.58 \pm 7.9$ & 0.747 \\
\hline TSH (uIU/ml) & $1.7 \pm 0.85$ & $1.74 \pm 1.04$ & 0.617 \\
\hline Horizontal Curvature - Right Eye (D) & $43.98 \pm 1.77$ & $43.68 \pm 1.76$ & 0.130 \\
\hline Vertical Curvature - Right Eye (D) & $44.22 \pm 1.82$ & $44.05 \pm 1.66$ & 0.361 \\
\hline Horizontal Curvature - Left Eye (D) & $44.03 \pm 1.74$ & $43.81 \pm 1.77$ & 0.377 \\
\hline Vertical Curvature - Left Eye (D) & $44.36 \pm 1.74$ & $44.1 \pm 1.74$ & 0.346 \\
\hline Astigmatism - Right Eye (D) & $0.55 \pm 0.46\left(3^{\circ}-176^{\circ}\right)$ & $0.61 \pm 0.49\left(1^{\circ}-180^{\circ}\right)$ & 0.494 \\
\hline Astigmatism - Left Eye (D) & $0.56 \pm 0.42\left(1^{\circ}-180^{\circ}\right)$ & $0.59 \pm 0.41\left(2^{\circ}-180^{\circ}\right)$ & 0.486 \\
\hline CCT - Right eye $(\mu \mathrm{m})$ & $549.94 \pm 44.13$ & $528.35 \pm 46.53$ & 0.017 \\
\hline CCT - Left eye $(\mu \mathrm{m})$ & $548.52 \pm 45.33$ & $527.06 \pm 47.01$ & 0.017 \\
\hline IOP - Right eye (mmHG) & $14.06 \pm 2.49$ & $14.69 \pm 2.95$ & 0.254 \\
\hline IOP - Left eye (mmHG) & $14.26 \pm 2.5$ & $14.5 \pm 2.81$ & 0.638 \\
\hline ST 1 - Right eye (mm) & $16.56 \pm 8.74$ & $11.26 \pm 6.8$ & 0.001 \\
\hline ST 1 - Left eye (mm) & $15.63 \pm 7.85$ & $11.22 \pm 6.8$ & 0.003 \\
\hline
\end{tabular}

SD: Standard deviation, D: Diopter, İOP: İntraocular pressure, CCT: Central corneal thickness, ST 1: Schirmer I test, n: number.

Table 2: Correlation between estradiol levels and horizontal and vertical curvatures, astigmatism values, CCT values, and Schirmer I test scores of both eyes.

\begin{tabular}{|lll|}
\hline Parameter & rs & p \\
\hline Right eye horizontal curvature (D) & 0.103 & 0.289 \\
\hline Left eye horizontal curvature (D) & 0.050 & 0.604 \\
\hline Right eye vertical curvature (D) & 0.006 & 0.953 \\
\hline Left eye vertical curvature (D) & 0.012 & 0.898 \\
\hline Right eye astigmatism (D) & -0.008 & 0.936 \\
\hline Left eye astigmatism (D) & -0.035 & 0.720 \\
\hline Right eye CCT $(\mu \mathrm{m})$ & 0.286 & 0.003 \\
\hline Left eye CCT $(\mu \mathrm{m})$ & 0.261 & 0.006 \\
\hline Right eye ST 1 $(\mathrm{mm})$ & 0.279 & 0.004 \\
\hline Left eye ST 1 $(\mathrm{mm})$ & 0.298 & 0.002 \\
\hline
\end{tabular}

D: Diopter, CCT: Central corneal thickness, ST 1: Schirmer I test

In particular, with the growing use of corneal refractive surgery, the importance of corneal thickness and agerelated ocular changes has been further increased. Kiely et al. found that horizontal and vertical curvatures steepen at the beginning of the menstrual cycle and flatten after ovulation and reported that estradiol was the main determiner of these changes. The authors also suggested that, through the menstrual cycle, corneal thickness increased with the increase in the estrogen level. ${ }^{10}$ Nevertheless, there are several studies suggesting that corneal thickness does not change throughout the menstrual cycle. ${ }^{11}$
Table 3: Correlation between progesterone levels and horizontal and vertical curvatures, astigmatism values, CCT values, and Schirmer I test scores of both eyes.

\begin{tabular}{|lll|}
\hline Parameter & $\mathrm{r}$ & $\mathrm{p}$ \\
\hline Right eye horizontal curvature (D) & 0.034 & 0.728 \\
\hline Left eye horizontal curvature (D) & 0.018 & 0.856 \\
\hline Right eye vertical curvature (D) & 0.012 & 0.898 \\
\hline Left eye vertical curvature (D) & 0.056 & 0.568 \\
\hline Right eye astigmatism (D) & -0.170 & 0.079 \\
\hline Left eye astigmatism (D) & -0.119 & 0.219 \\
\hline Right eye CCT $(\mu \mathrm{m})$ & 0.146 & 0.131 \\
\hline Left eye CCT $(\mu \mathrm{m})$ & 0.165 & 0.088 \\
\hline Right eye ST $1(\mathrm{~mm})$ & 0.202 & 0.036 \\
\hline Left eye ST 1 $(\mathrm{mm})$ & 0.194 & 0.044 \\
\hline
\end{tabular}

D: Diopter, CCT: Central corneal thickness, ST 1: Schirmer I test

Table 4: Correlation between estradiol and progesterone levels and dry eye scores (Kruskal Wallis H-Test).

\begin{tabular}{|l|ll|l|}
\hline & Mean & SD & p \\
\hline Estradiol & 57.03 & 67.88 & 0.009 \\
\hline Progesterone & 3.32 & 4.99 & 0.118 \\
\hline
\end{tabular}

SD: Standard deviation

On the other hand, it has also been hypothesized that corneal thickness also increases during pregnancy when the estrogen and progesterone levels show a significant 
increase, and that this increase is mainly associated with estrogen. ${ }^{12}$ Although there is little documentation about the CCT changes in postmenopausal women, SanchisGimeno JA et al. demonstrated that CCT was lower in postmenopausal women with dry eye symptoms compared to postmenopausal women without dry eye symptoms. ${ }^{13}$ This finding suggests that, in postmenopausal women, dry eye may also has an additional effect on the decrease in CCT. A randomized controlled study investigating menopausal women indicated that no significant corneal curvature changes were detected between premenopausal and postmenopausal women and estrogen established a significant negative correlation with horizontal curvature in postmenopausal women but estrogen did not correlate with the corneal curvature changes in premenopausal women and progesterone did not correlate with the corneal curvature changes both in pre- and postmenopausal women. ${ }^{14}$

In the present study, we also found that CCT significantly decreased in postmenopausal women, estradiol established a positive correlation with corneal thickness, CCT decreased as the estrogen level decreased, and there was no correlation between the progesterone level and CCT. However, no significant differences were observed between premenopausal and postmenopausal women with regards to horizontal and vertical curvatures and astigmatism values of both eyes. Depending on these findings, we follow the hypothesis proposed by Wagner $\mathrm{H}$ et al., which suggested that topographic changes may occur in the eye in response to the loss of sex steroid hormones but these have little or no significance in clinical care. $^{4}$

Dry eye remains a major challenge in postmenopausal women, particularly in advanced-age women. Dry eye disease leads to a number of symptoms that cause adverse outcomes for the patient's quality of life, including ocular dryness, burning, foreign body sensation, redness in the eyes, blurred vision, watery eyes, eye irritation when reading or driving, ocular pain when waking, photophobia, and sensitivity against environmental factors such as air conditioner or windy conditions. ${ }^{15}$ Ocular tissue is considered to be a target organ for sex steroid hormones since there are estrogen, progesterone, and androgen receptor mRNAs which have been shown to translate into receptor proteins in the lacrimal gland, meibomian gland, lid, palpebral and bulbar conjunctivae, cornea, iris and ciliary body, lens, and retina in rats, rabbits, or humans. The sex steroid hormones exert their effect possibly by influencing gene expression through other pathways and by eliciting acute responses that affect ocular surface homeostasis., ${ }^{1,2}$ The loss of sex steroid hormones in the postmenopausal term may lead to dry eye by causing reduced corneal thickness as well as alterations in homeostasis and in the layers of the tear film. Structural and functional weakening of lacrimal and meibomian glands, attributed to menopause, and significant thinning of the lipid and aqueous layers, as a result of with hyposecretion, may result in higher rates of tear evaporation, thus leading to increased tear film osmolarity. In turn, this hyperosmolarity activates inflammatory cascades in the cornea and conjunctiva, leading to the activation of nociceptor signals by inflammatory mediators and thus resulting in subjective symptoms of dry eye. ${ }^{15}$

The relation of DES and sex-hormone levels has driven attention in recent studies. Gagliano et al. found that the $17-\beta$-estradiol, estrone, and total testosterone levels were lower in postmenopausal women with DES compared to controls and these hormone levels were negatively correlated with tear osmolarity. ${ }^{16}$ A prospective study revealed that the administration of estradiol patch after surgical menopause for 12 months reduced tear production and decreased the mean Schirmer test score by $20 \%$, whereas a randomized controlled study indicated that the administration of phytoestrogen therapy in postmenopausal women decreased tear osmolarity and increased tear production. ${ }^{17,18}$ This might be revealing that spontaneous menopause with its gradually decreasing estrogen and aging are more important factors for tear production. Although several studies reported that combined estrogen and progesterone therapy increased tear flow in postmenopausal women, some other studies showed that there is no effect on tear flow, or even a decrease in tear production. ${ }^{15}$ In the present study, the ST 1 scores in the postmenopausal women were significantly lower for both eyes. It was also revealed that the estradiol and progesterone levels were positively correlated with the ST 1 scores for both eyes, and that tear production was negatively affected by the decrease in estradiol and progesterone levels. Moreover, the analysis of the relationship between the estradiol and progesterone levels and dry eye scores revealed that the dry eye scores increased with the decrease in estradiol levels but were not affected by the decrease in progesterone levels. Depending on these findings, we postulate that the estrogen and progesterone are associated with dry eye in postmenopausal women but their exact effect mechanism remains controversial, and that these hormones may affect dry eye symptoms in a multifactorial manner, by influencing not only the secretion amounts of lacrimal and sebaceous glands but also with their content and osmolarity as well as the immune inflammatory processes and the structure of the ocular surface.

Our study was limited by the small number of patients. Therefore, future studies with larger patient series that will involve pre- and post-menopausal women and longterm follow-up periods extending from the premenopausal term to the postmenopausal term are needed.

\section{CONCLUSION}

In conclusion, decreased estrogen levels are associated with CCT and dry-eye symptoms, decreased estrogen and progesteron levels correlates well with diminished tear 
production but progesterone levels does not affect dryeye symptoms. The ocular surface disorders in postmenopausal women, particularly dry eye syndrome, can be regarded as endocrinal disorders and thus a multidisciplinary approach involving ophthalmologists and gynecologists could be useful for their treatments. In patients with dry eye, excessive thinning of the cornea may affect the refractive surgery of the cornea; therefore, preoperative measurement of corneal thickness should be performed in these patients.

Funding: No funding sources Conflict of interest: None declared

Ethical approval: The study was approved by the Institutional Ethics Committee

\section{REFERENCES}

1. Suzuki T, Kinoshita Y, Tachibana M, Matsushima Y, Kobayashi Y, Adachi W, et al. Expression of sex steroid hormone receptors in human cornea. Curr Eye Res. 2001;22(1):28-33.

2. Wickham LA, Gao J, Toda I, Rocha EM, Ono M, Sullivan DA. Identification of androgen, estrogen and progesterone receptor mRNAs in the eye. Acta Ophthalmol Scand. 2000;78(2):146-53.

3. Sullivan DA, Edwards JA, Wickham LA, Pena JD, Gao J, Ono M, et al. Identification and endocrine control of sex steroid binding sites in the lacrimal gland. Current Eye Research, Curr Eye Res. 1996;15(3):279-91.

4. Brewitt H, Sistani F. Dry eye disease: the scale of the problem. Surv Ophthalmol. 2001;45(2):199-202.

5. Schaumberg DA, Sullivan DA, Buring JE, Dana MR. Prevalence of dry eye syndrome among US women. Am J Ophthalmol. 2003;136(2):318-26.

6. Lemp MA, Bron AJ, Baudouin C, Benítez Del Castillo JM, Geffen D, Tauber J, et al. Tear osmolarity in the diagnosis and management of dry eye disease. Am J Ophthalmol. 2011;151(5):792-98.

7. Sullivan DA, Wickham LA, Rocha EM, Kelleher RS, da Silveira LA, Toda I. Influence of gender, sex steroid hormones and the hypothalmic-pituitary access on the structure and function of the lacrimal gland. Adv Exp Med Biol. 1998;438:11-42.

8. Sullivan DA, Yamagami H, Liu M, Steagall RJ, Schirra F, Suzuki T, et al. Sex steroids, the meibomian gland and evaporative dry eye. Adv Exp Med Biol. 2002;506(Pt A):389-99.

9. Gipson IK. Age-related changes and diseases of the ocular surface and cornea. Invest Ophthalmol Vis Sci. 2013;54(14):ORSF48-53.

10. Kiely PM, Carney LG, Smith G. Menstrual cycle variations of corneal topography and thickness. Am J Optom Physiol Opt. 1983;60(10):822-9.

11. Wagner H, Fink BA, Zadnik K. Sex- and genderbased differences in healthy and diseased eyes. Optometry. 2008;79(11):636-52.

12. Weinreb RN, Lu A, Beeson C. Maternal corneal thickness during pregnancy. Am J Ophthalmol. 1988;105(3):258-60.

13. Sanchis-Gimeno JA, Lleó-Pérez A, Alonso L, Rahhal MS, Martínez-Soriano F. Reduced corneal thickness values in postmenopausal women with dry eye. Cornea. 2005;24(1):39-44.

14. Aydin E, Demir HD, Demirturk F, Caliskan AC, Aytan H, Erkorkmaz U. Corneal topographic changes in premenopausal and postmenopausal women. BMC Ophthalmol. 200;7:9.

15. Sriprasert I, Warren DW, Mircheff AK, Stanczyk FZ. Dry eye in postmenopausal women: a hormonal disorder. Menopause. 2016;23(3):343-51.

16. Gagliano C, Caruso S, Napolitano G, Malaguarnera G, Cicinelli MV, Amato R, et al. Low levels of 17betaoestradiol, oestrone and testosterone correlate with severe evaporative dysfunctional tear syndrome in postmenopausal women: a case-control study. Br J Ophthalmol. 2014;98(3):371-6.

17. Uncu G, Avci R, Uncu Y, Kaymaz C, Develioğlu O. The effects of different hormone replacement therapy regimens on tear function, intraocular pressure and lens opacity. Gynecol Endocrinol. 2006;22(9):501-5.

18. Scuderi G, Contestabile MT, Gagliano C, Iacovello D, Scuderi L, Avitabile T. Effects of phytoestrogen supplementation in postmenopausal women with dry eye syndrome: a randomized clinical trial. Can $\mathrm{J}$ Ophthalmol. 2012;47(6):489-92.

Cite this article as: Turgut FG, Turgut A, Dolgun ZN, Köroğlu N. Effects of menopause on corneal topography and dry eye. Int J Reprod Contracept Obstet Gynecol 2017;6:461-5. 